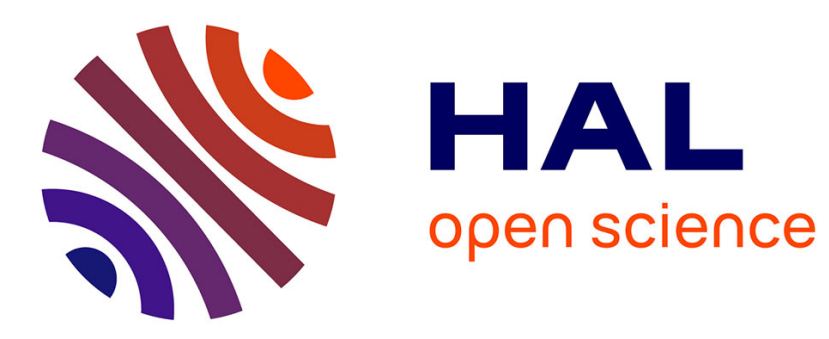

\title{
Experimental and theoretical studies of a dual-frequency laser free from anti-phase noise
}

\author{
Abdelkrim El Amili, Syamsundar De, Goulc'Hen Loas, Fabien Bretenaker, \\ Mehdi Alouini
}

\section{- To cite this version:}

Abdelkrim El Amili, Syamsundar De, Goulc'Hen Loas, Fabien Bretenaker, Mehdi Alouini. Experimental and theoretical studies of a dual-frequency laser free from anti-phase noise. Advanced SolidState Lasers Congress, Oct 2013, Paris, France. pp.ATu3A.52, 10.1364/ASSL.2013.ATu3A.52 . hal01044902

\section{HAL Id: hal-01044902 \\ https://hal.science/hal-01044902}

Submitted on 24 Jul 2014

HAL is a multi-disciplinary open access archive for the deposit and dissemination of scientific research documents, whether they are published or not. The documents may come from teaching and research institutions in France or abroad, or from public or private research centers.
L'archive ouverte pluridisciplinaire HAL, est destinée au dépôt et à la diffusion de documents scientifiques de niveau recherche, publiés ou non, émanant des établissements d'enseignement et de recherche français ou étrangers, des laboratoires publics ou privés. 


\title{
Experimental and theoretical studies of a dual-frequency laser free from anti-phase noise
}

\author{
A. El Amili, S. De ${ }^{2}$, G. Loas ${ }^{1}$, F. Bretenaker ${ }^{2}$, and M. Alouini ${ }^{1}$ \\ 1. Institut de Physique de Rennes, Université de Rennes 1, CNRS, Campus de Beaulieu, 35042 Rennes, France \\ 2. Laboratoire Aimé-Cotton, CNRS-Université Paris 11, 91405 Orsay Cedex, France \\ Abdelkrim.elamili@univ-rennes1.fr
}

\begin{abstract}
Strong reduction of the anti-phase intensity noise is shown in a two-polarization dualfrequency solid-state laser. The spectral behavior of the intensity noise correlations between the two orthogonally polarized modes is investigated, both experimentally and theoretically.

OCIS codes: (140.0140) Lasers and laser optics, (140.3530) Lasers, neodymium; (140.3580) Lasers, solid-state; (270.2500)

Fluctuations, relaxations, and noise
\end{abstract}

\section{Introduction}

Dual-frequency solid-state lasers are attractive for a large number of applications such as microwave photonics, spectroscopy, and metrology. The main limitation for these lasers is the high intensity noise at low frequencies (few $\mathrm{kHz}$ to few $\mathrm{MHz}$ ) coming from the relaxation oscillations since these lasers are of class-B type. As dual-frequency lasers are concerned, the simultaneous oscillation of two partially coupled modes leads to the existence of two noise peaks in the intensity noise spectrum, corresponding to the so-called in-phase and anti-phase relaxation oscillation frequencies. The in-phase noise, which corresponds to the standard relaxation oscillations of the laser, can be reduced either electronically or optically using feedback loops [1]. However, the anti-phase noise, which is related to a resonant exchange of energy between the two laser modes, is very difficult to circumvent because the reduction of this noise would require an additional servo-loop acting on the difference of the intensities of the two modes or two servo-loops acting independently on the intensity of each mode. An optimal dual-frequency laser in terms of intensity noise and beat frequency stability would be a single axis laser in which the population inversions related to each mode are independent. Here, we demonstrate experimentally and theoretically how the proper design of a two polarization dual-frequency solid-state laser allows to get rid of the anti-phase noise in the simplest possible architecture and without using any electronic or optical feedback loop. This design is based on an appropriate choice of the active medium cut and orientation in order to assign two almost independent families of active atoms to the two laser modes. The nonlinear coupling constant in this laser being adjustable, we present how the spectral correlation between the intensity noises of the two orthogonally polarized modes behaves according to the coupling constant.

\section{Antiphase noise reduction}

(001)

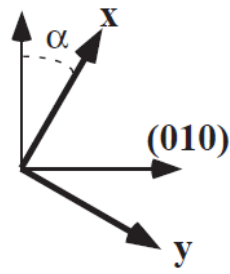

a)

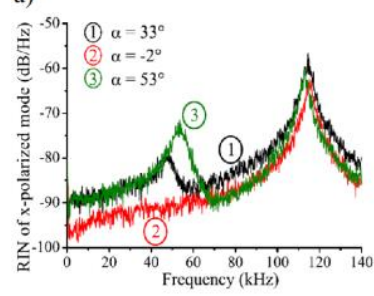

b)

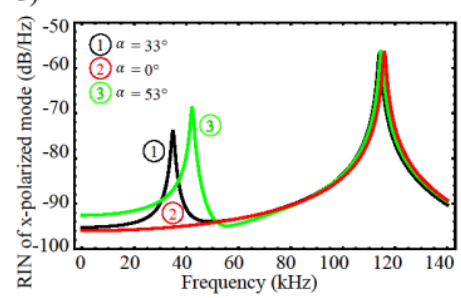

Fig. 1. a) Experimental RIN spectra observed when only one of the two laser modes is detected, for three values of $\alpha$. b) Corresponding theoretical RIN spectra computed with the following parameters; excitation ratios for the two modes: $r_{x}=1.55$ and $r_{y}=1.5$; cavity decay rates: $\gamma_{\mathrm{x}}=5.36 \times 107 \mathrm{~s}^{-1}$ and $\gamma_{\mathrm{y}}=\gamma_{\mathrm{x}} \mathrm{r}_{\mathrm{x}} / \mathrm{r}_{\mathrm{y}}$; population inversion lifetime: $1 / \Gamma=200 \mu \mathrm{s}$; pump $\mathrm{RIN}=-105 \mathrm{~dB} / \mathrm{Hz}$; level of correlation between pump fluctuations $\eta=0.95$.

It is has recently been shown that, in Nd:YAG, the emitting dipoles behave as if they were aligned along the crystallographic axes of the matrix [2]. In particular, by choosing a (100)-cut Nd:YAG crystal instead of the more common (111) cut, it was demonstrated that almost complete decoupling of two perpendicularly polarized laser modes could be obtained by aligning them with the (010) and (001) crystallographic axes. One then expect the antiphase noise to vanish using a properly oriented (100)-cut Nd:YAG crystal. In order to adjust the angle, $\alpha$, between the laser polarization eigen-states and the crystallographic axes of the active medium, we insert into the two-mirror cavity a birefringent plate whose neutral axes define the two crossed and linear polarizations of the laser. Fig. 1(a) reproduces the relative intensity noise (RIN) spectra recorded when only the x-polarized mode is detected for three 
values of $\alpha$. For $\alpha=33^{\circ}$ and $\alpha=53^{\circ}$, we observe the existence of the usual in-phase relaxation oscillation peak at $115 \mathrm{kHz}$ and of the anti-phase relaxation oscillation peak at about 50 to $60 \mathrm{kHz}$ whose amplitude depends on the value of $\alpha$. Now, we rotate $\alpha$ while detecting only the x-polarization and look for a position in which the amplitude of the anti-phase peak is minimized. This leads to $\alpha=-2^{\circ}$ and to the red spectrum in Fig. 1(a). As expected, for this orientation which is close to $\alpha=0^{\circ}$ and for which the coupling is expected to be minimum [2], the anti-phase peak becomes so small that it disappears below the noise floor.

These previous results can be predicted theoretically from a model based on the following rate equations [2]:

$$
\begin{gathered}
\frac{d F_{x}}{d t}=-\gamma_{x} F_{x}+\kappa F_{x}\left[N_{1} A(\alpha)+N_{2} B(\alpha)\right], \\
\frac{d F_{y}}{d t}=-\gamma_{y} F_{y}+\kappa F_{y}\left[N_{2} A(\alpha)+N_{1} B(\alpha)\right], \\
\frac{d N_{1}}{d t}=\Gamma\left(N_{01}-N_{1}\right)-\kappa N_{1}\left[F_{x} A(\alpha)+F_{y} B(\alpha)\right], \\
\frac{d N_{2}}{d t}=\Gamma\left(N_{02}-N_{2}\right)-\kappa N_{2}\left[F_{x} B(\alpha)+F_{y} A(\alpha)\right],
\end{gathered}
$$

where $F_{x}$ and $F_{y}$ are the photon numbers corresponding to the $x$ - and y-polarized modes, respectively. $\gamma_{x}$ and $\gamma_{y}$ are their corresponding cavity decay rates. $\mathrm{N}_{1}$ and $\mathrm{N}_{2}$ are the population inversions for the two families of dipoles. Their lifetime is $1 / \Gamma$ and their pumping rates are modeled by $\mathrm{N}_{01}$ and $\mathrm{N}_{02} . \kappa$ is proportional to the stimulated emission cross section, and the coupling between the two laser modes and the two families of dipoles is taken into account in the coefficients $\mathrm{A}$ and $\mathrm{B}$ which depend on the orientation $\alpha$. The intensity noise spectra of each mode can be found by introducing pump noise fluctuations in the pumping terms $\mathrm{N}_{01}$ and $\mathrm{N}_{02}$, with an adjustable level of correlation $\eta$ between the pump fluctuations entering the two families of dipoles. This leads to the theoretical noise spectra for the x-polarized mode which are reproduced in Fig. 1(b). These spectra have been computed with the values of the different parameters corresponding to the experimental data of Fig. 1(a). We see that the theoretical results are in excellent agreement with the experimental data. The anti-phase peak which are present for $\alpha=33^{\circ}$ and $\alpha=53^{\circ}$ almost completely disappears for $\alpha=-2^{\circ}$, just like in Fig. 1(a). Finally, the computed evolution of the amplitude and frequency of the anti-phase peak with $\alpha$ is consistent with the experimental results.

\section{Intensity noise correlations}

When one considers the application of such sources to microwave photonics, it is worth remembering that the spectral purity of the RF-beat note also depends on the correlation of the fluctuations of the two oscillating modes. Now, our aim is to study the correlation between the intensity noises of the two orthogonally polarized modes of the Nd:YAG laser. The noise correlations between of the two oscillating modes can be analyzed through the normalized correlation spectrum which is defined as:

$$
\Theta(f)=\frac{\left\langle\delta \widetilde{F}_{x}(f) \delta \widetilde{F}_{y}^{*}(f)\right\rangle}{\sqrt{\left\langle\left.\delta \widetilde{F}_{x}(f)\right|^{2}\right\rangle\left\langle\delta \widetilde{F}_{y}^{*}(f)^{2}\right\rangle}} .
$$

We define the degree of the intensity spectral correlation as $|\Theta(f)|^{2}$ (where $0<|\Theta(f)|<1$ ) and the correlation phase spectrum as $\operatorname{Arg}[\Theta(\mathrm{f})]$.

Fig. 2 reproduces the results obtained for $\alpha=30^{\circ}$, which corresponds to a moderate coupling between the two modes (coupling constant equal to 0.4). The experimental and theoretical RIN spectra for the two laser modes are shown in Figs. 2(a) and 2(b), respectively. In the experimental RIN spectra (Fig. 2(a)), we find the in-phase peak at about $90 \mathrm{kHz}$ and anti-phase peak at about $50 \mathrm{kHz}$. The RIN spectra for the two modes are again slightly different, since the intra-cavity losses and gains are different. The corresponding theoretical RIN spectra (Fig. 2(b)) show good agreement with the experimental apart from the fact that the anti-phase peak is at about $40 \mathrm{kHz}$ (Fig. 2(b)), although experimentally we find it at about $50 \mathrm{kHz}$ (Fig. 2(a)). The experimentally measured noise correlation intensity and phase spectra are shown in Figs. 2(c) and 2(e), respectively. For these experiments, a polarization beam splitter enables to acquire simultaneously the intensities along $\mathrm{x}$ and $\mathrm{y}$ polarizations with two identical but separate photodiodes. The obtained experimental spectra show the existence of two dips in the correlation intensity spectrum at frequencies of about $25 \mathrm{kHz}$ and $55 \mathrm{kHz}$, as shown in Fig. 2 (c). Moreover, we also see that the correlation intensity reaches $0 \mathrm{~dB}$ once at about $50 \mathrm{kHz}$, again at $70 \mathrm{kHz}$ and maintains $0 \mathrm{~dB}$ value for all higher frequencies. 

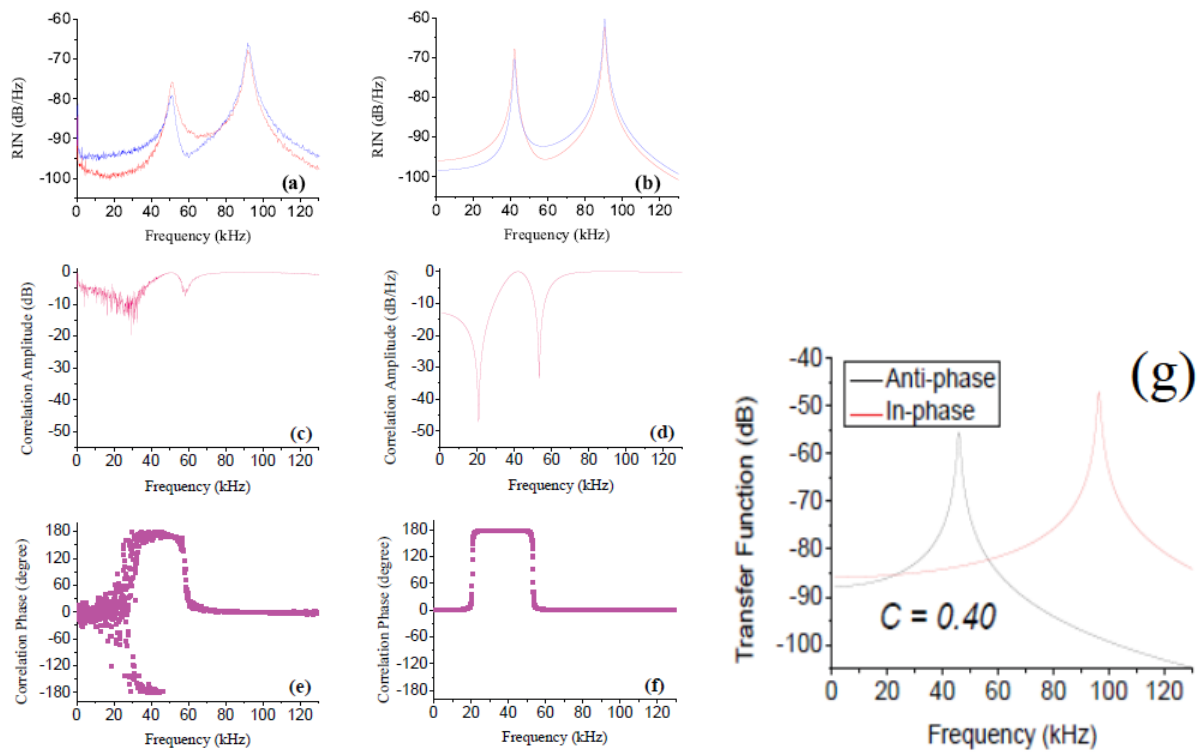

Fig. 2. Results for $\alpha=30^{\circ}$, which corresponds to a coupling constant value $\mathrm{C}=0: 40$. (a) Experimental and (b) theoretical RIN spectra for the two oscillating modes (red and blue plots correspond to $\mathrm{x}$ - and y-polarized modes, respectively); (c) experimental and (d) theoretical intensity noise correlation amplitude spectra; (e) experimental and (f) theoretical intensity noise correlation phase spectra. (g) Normalized transfer functions for the in-phase (red line) and anti-phase (black line) relaxation oscillation mechanisms

Now in the experimental correlation phase spectrum (Fig. 2.(e)), we find two jumps, one from 0 to $\pi$ at about 25 $\mathrm{kHz}$ and the other one at about $55 \mathrm{kHz}$ from $\pi$ to 0 . Moreover, the correlation phase is 0 for frequencies in between 0 $-25 \mathrm{kHz}$ and also for frequencies higher than $55 \mathrm{kHz}$, whereas it is $\pi$ within $25-55 \mathrm{kHz}$. The theoretical correlation intensity and phase spectra, presented in Figs. 2(d) and 2(f) respectively, show satisfactory agreement with the experiment (Figs. 2(c) and 2(e) respectively). The dips on the correlation intensity spectra correspond to phase jumps in the correlation phase spectra. These phase jumps correspond to the crossings between the responses of the in-phase and anti-phase relaxation mechanisms (Fig. 2 (g)). Moreover, the fact that the correlation amplitude is rather low for frequencies lower than $20 \mathrm{kHz}$, comes from the facts that i) the pump noises are only partially correlated $(\eta<1)$ and ii) the amplitude of the transfer functions of the in-phase and anti-phase mechanisms are very close in this region leading to low intensity correlation. By contrast, in the high frequency region (above $60 \mathrm{kHz}$ ), the two transfer functions are separated by several orders of magnitude and the intensity correlation becomes very close to 1 .

\section{Conclusion}

We show that the noise induced by the anti-phase relaxation oscillations resonance in a dual-frequency laser can be almost completely cancelled by a proper choice the active medium and design of the cavity. This has been illustrated in the case of a Nd:YAG crystal in which the choice of a (100)-cut crystal together with the proper orientation of the polarizations of the laser modes permit to cancel this resonance by more than $20 \mathrm{~dB}$ [3]. Besides, the coupling constant between the two modes being easily adjustable in this laser, we have investigated both experimentally and theoretically the behaviour of the correlation between the intensity noises of the two orthogonally polarized modes. Since the dual-frequency Nd:YAG laser is a class-B type, the present study generalizes our previous work on noise correlations in dual-frequency class-A VECSEL [4] which is free from relaxation oscillations.

\section{References}

[1] S. Taccheo, P. Laporta, O. Svelto, and G. de Geronimo, "Intensity noise reduction in a single-frequency ytterbium-codoped erbium laser," Opt. Lett. 21, 1747-1749 (1996).

[2] S. Schwartz, G. Feugnet, M. Rebut, F. Bretenaker, and J. P. Pocholle, "Orientation of $\mathrm{Nd}^{3+}$ dipoles in yttrium aluminium garnet: experiment and model," Phys. Rev. A 79, 063814 (2009).

[3] A. El Amili, G. Loas, S. De, S. Schwartz, G. Feugnet, J. P. Pocholle, F. Bretenaker, and M. Alouini, "Experimental demonstration of a dualfrequency laser free from antiphase noise, ” Opt. Lett. 37, 4901-4903 (2012).

[4] S. De, V. Pal, A. El Amili, G. Pillet, G. Baili, M. Alouini, I. Sagnes, R. Ghosh, and F. Bretenaker, "Intensity noise correlations in a twofrequency VECSEL, "Opt. Express 21, 2538-2550 (2013). 